\title{
Spiral Waves Generation using an Eikonal-reaction Cardiac Electrophysiology Model
}

\author{
Narimane Gassa ${ }^{1,2,3}$, Nejib Zemzemi ${ }^{1,2,3}$, Cesare Corrado ${ }^{4}$, and Yves \\ Coudière ${ }^{1,2,3}$ \\ 1 University of Bordeaux, IMB, Bordeaux, France \\ 2 National Institue of Mathematics and Informatics, Inria Bordeaux, France \\ 3 IHU-Lyric, Bordeaux, France \\ 4 School of Biomedical Engineering and Imaging Sciences, Kings College London, \\ London, UK.
}

\begin{abstract}
Aim: Computer models enabled the study of the fundamental mechanisms responsible for arrhythmias and have the potential of optimizing the clinical procedure for an individual patients pathology. The model complexity and the computational costs affecting computer models hamper their application on a routinely performed procedure. In this work, we aim to design a computer model suitable for clinical time scales. Methods: We adopt a (multi-front) eikonal model that adapts the conduction velocity to the underlying electrophysiology; we describe the diffusion current using a parametrised form, fitted to reproduce the monodomain profile. Results: We simulated spiral waves on a $3 \mathrm{D}$ tissue slab and bi-atrial anatomy. We compared the numerical results obtained with a monodomain formulation with those obtained with the new method. Both models provided the same pattern of the spiral waves. While the monodomain model presented slower propagation fronts, the eikonal model captured the correct value of the conduction velocity $\mathrm{CV}$ even using a coarse resolution. Conclusion: The eikonal model has the potential of enabling computer-guided procedures when adapts the conduction velocity to the underlying electrophysiology and characterises the diffusion current with a parametrised form.
\end{abstract}

Keywords: Cardiac Electrophysiology · Eikonal model · Spiral Wave · Atrial fibrillation.

\section{Introduction}

Cardiac ablation is widely used to treat cardiac atrial arrhythmias, and sometimes also ventricular arhythmias. In these heart conditions, the heartbeat could be slow in the case of bradycardia and it could be fast in cases of tachycardia, flutter or fibrillation. In these latter conditions, cardiac ablation techniques are used in order to restore a normal heart rhythm. This technique allows isolating trigger areas from the rest of the heart. In order to accurately identify the regions 
to ablate, catheter are introduced inside the heart chambers and measurements of the electrical signals allow to localize the abnormal regions. This invasive method is performed in the operating room where the patient is under local anesthesia. It is long and sometimes inaccurate, mainly because of the heart movement. The recent alternative to this approach is the non invasive electrocardiography imaging (ECGI), this method allows to reconstruct electrical informations on the heart surface using a set of electrodes measuring the electrical potential on the body surface and geometrical information about the patient extracted from CT-scans [10]. In this method an unstable mathematical inverse problem is solved and the results are not very satisfactory in clinical application [3]. One of the approaches, that could have a high potential to solve the ECGI inverse problem is to use propagation models in the cardiac domain and parametrize these models in order to personalize the model by fitting it to the electrical measurements. During the last decades, advances have been made in electrocardiology. The bidomain and monodomain models [1] are widely used as they are considered to accurately describe the electrical propagation in the heart. In spite of being applied successfully, these phenomenological models based on reactiondiffusion equations are known to be computationally very expensive. Thus their use in clinical application is challenged and non-practical. The eikonal equation, on the other hand, is a one non-linear partial differential equation that arises in problems of wave propagation and can be very fast to compute. Despite its low computational complexity, the eikonal model is less reliable in arrhythmia prediction than the reference models, which can be a major limit for studying arrhythmias. In particular, in the literature many studies have been dedicated to the use of the eikonal model in cardiac electrophysiology $[4,6,2]$. In Neic et al (2017) [6] a new reaction-eikonal model has been introduced, but, as the authors stated in the discussion, this model is not suitable for reentries. In all these studies no spiral waves have been generated with the different formulations of eikonal models. This is mainly due to the fact that the eikonal models provides the activation times in the depolarization phase but does not accurately describe the repolorization of the cardiac cells. In this work, we present a novel approach wherein we enhance the conduction velocity adapted eikonal model (EK-CV) presented in [2] with a time-varying parametric function that describes the diffusive current. We then fit this expression to the diffusive current we obtained by solving the monodomain model. This new formulation allows the EK-CV model to simulate the dynamics of the spiral waves. We compare the generated waves with those simulated using the monodomain model.

\section{Methods}

\subsection{The Monodomain Mitchell-Schaeffer model}

The Mitchell-Schaeffer (MS) ionic model [5] describes the ionic currents that are flowing through the cell membrane. It is characterised by two state variables, the transmembrane voltage $V_{m}$ and a gating variable $h$. Given a cardiac domain 
$\Omega \subset \mathbb{R}^{d}(d=2,3)$ the mondomain in $\Omega$ coupled with the MS model can be written as follows:

$$
\begin{cases}A_{m}\left(C_{m} \partial_{t} V_{m}+\beta I_{\text {ion }}\left(V_{m}, h\right)\right)-\operatorname{div}\left(\underline{\underline{D}}_{m} \underline{\nabla} V_{m}\right)=A_{m} I_{a p p}, & \text { in } \Omega \times \mathbb{R}^{+}, \\ \partial_{t} h+g\left(V_{m}, h\right)=0, & \text { in } \Omega \times \mathbb{R}^{+}, \quad(1) \\ \underline{\underline{D}}_{m} \nabla V_{m} \cdot n=0, & \text { on } \partial \Omega\end{cases}
$$

with an ionic model given by,

$$
\begin{array}{r}
I_{\text {ion }}\left(V_{m}, h\right)=-\frac{h V_{m}^{2}\left(1-V_{m}\right)}{\tau_{\text {in }}}+\frac{V_{m}}{\tau_{\text {out }}} \\
g\left(V_{m}, h\right)= \begin{cases}\frac{h-1}{\tau_{\text {open }}}, & \text { if } V_{m} \leq V_{\text {gate }} \\
\frac{h}{\tau_{\text {close }}}, & \text { if } V_{m}>v_{\text {gate }}\end{cases}
\end{array}
$$

\subsection{The EK-CV model}

The eikonal model in cardiac electrophysiology has been proposed in the early nineties [4] as an alternative to the reaction-diffusion bidomain to compute the activation time $T_{\text {act }}(x)$ representing the arrival of the depolarization wavefront at each point $x \in \Omega$. The activation time $T_{a c t}(x)$ solves the eikonal equation:

$$
\begin{cases}F \sqrt{\left(\underline{\nabla} T_{a c t}\right)^{T} \underline{D} \underline{\nabla} T_{a c t}}=1, & \text { on } \Omega, \\ T_{a c t}=0, & \text { on } \Gamma,\end{cases}
$$

where $\underline{\underline{D}}$ is a dimensionless tensor that describes the tissue anisotropy, the scalar function $F$ is the velocity of the front and $\Gamma$ is the set of the first activated sites. The EK-CV model [2] makes the conduction velocity $F$ dependent on the underlying electrical state as follows:

$$
\begin{gathered}
F=\alpha \sqrt{\frac{2 \sigma_{m}^{l}}{\tau_{\text {in }}}}, \\
\alpha\left(D I_{n}\right)=\frac{1}{4}\left(3 \sqrt{h\left(D I_{n}\right)-h_{\min }}-\sqrt{h\left(D I_{n}\right)}\right) .
\end{gathered}
$$

Here $D I_{n}$ stands for the diastolic interval at the $n$-th beat and $\sigma_{m}$ is the diffusivity tensor, defined as follows:

$$
\sigma_{m}=\frac{\underline{\underline{D}}_{m}}{A_{m} C_{m}}
$$

with $\sigma_{m}^{l}$ is the longitudinal component parallel to the fibre direction $\vec{a}$ and $\sigma_{m}^{t}$ is the transverse component:

$$
\sigma_{m}=\sigma_{m}^{t} \underline{\underline{I}}+\left(\sigma_{m}^{l}-\sigma_{m}^{t}\right) \vec{a} \otimes \vec{a}=\sigma_{m}^{l}\left(\sigma_{m}^{t} / \sigma_{m}^{l} \underline{\underline{I}}+\left(1-\sigma_{m}^{t} / \sigma_{m}^{l}\right) \vec{a} \otimes \vec{a}\right)=\sigma_{m}^{l} \underline{\underline{D}} .
$$


Once discretised in time, within each time interval, we first evaluate $F$ using (3); next, we solve equation (2) using the fast marching method [9] and finally, we solve the ode system of the MS model:

$$
\begin{cases}\left.\partial_{t} V_{m}+I_{\text {ion }}\left(V_{m}, h\right)\right)=I_{\text {stim }}, & \text { in } \Omega \times \mathbb{R}^{+}, \\ \partial_{t} h+g\left(V_{m}, h\right)=0, & \text { in } \Omega \times \mathbb{R}^{+},\end{cases}
$$

where the function $I_{\text {ion }}$ and $g$ are the same functions as in the MS ionic model and the stimulation current $I_{\text {stim }}$ is computed using the activation time information and will be detailed in the following section.

\subsection{Parametrization of the diffusion current}

The method proposed in [2] describes the diffusive current with a stimulus of constant intensity and a given duration. This simple description of the diffusive term limits the study of spiral waves. In this work, we characterise the diffusive current with a time-varying parametric function, and we constrain the characterising parameters by fitting the diffusive term of the monodomain model. We parameterise the profile of the diffusive current with the sum of two Gaussian functions:

$$
I_{\text {stim }}(t)=A_{1} \cdot \exp -\frac{\left(t-\mu_{1}\right)^{2}}{2 \sigma_{1}^{2}}-A_{2} \cdot \exp -\frac{\left(t-\mu_{2}\right)^{2}}{2 \sigma_{2}^{2}}
$$

where the parameters $A_{1}, A_{2}$ are the magnitudes, $\mu_{1}, \mu_{2}$ are the times representing the centers of each Gaussian bump and $\sigma_{1}, \sigma_{2}$ are the standard deviations of the time in the Gaussian functions. The profile of (7) is shown in Figure $1 \mathrm{~B}$ and is consistent with the profiles obtained from the monodomain model simulations (Figure $1 \mathrm{~A}$ ). We post-processed the diffusive current from the monodomain model simulations as follows:

$$
\operatorname{div}\left(\underline{\underline{D}}_{m} \underline{\nabla} V_{m}\right)=I_{\text {stim }}=C_{m} \partial_{t} V_{m}+I_{\text {ion }} .
$$

and we centred around the activation time evaluated as the instant the action potential exceeds the threshold of 0.15 with a positive slope. Finally, we fit the function (7) to the mean value of all diffusion the currents shown in Fig. $1 \mathrm{~A}$ using a Levenberg-Marquardt algorithm [7] (Fig. $1 \mathrm{~A}$ ). We obtained $A_{1}=0.126, A_{2}=$ $0.143, \mu_{1}=1.81 \mathrm{~ms}, \mu_{2}=3.83 \mathrm{~ms}, \sigma_{1}=2.499, \sigma_{2}=2.188$ with relative error on the fitting of the mean value of the diffusion current is equal to 0.028 . In this work, inspired by the findings of Neic et al. (2017) [6], the stimulation current that will be introduced to the MS EK-CV model is the approximation of the diffusion current relative to a R-D monodomain model during the depolarization. $I_{\text {stim }}$ will be added, in space and in time, as follows:

$$
I_{\text {stim }}(t, x)= \begin{cases}I_{\text {stim }}(t), & \text { if } \mathrm{t} \in\left[T_{\text {act }}(x)-t_{\text {stim }}, T_{\text {act }}(x)+t_{\text {stim }}\right] \\ 0, & \text { else. }\end{cases}
$$




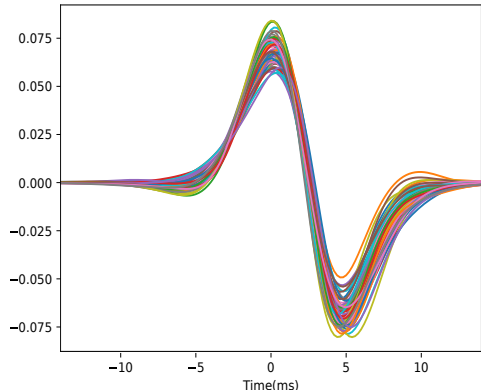

A

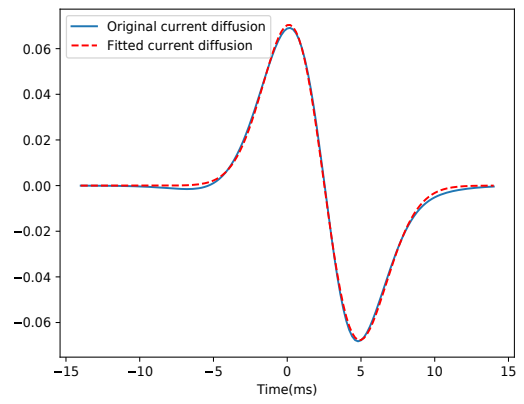

B

Fig. 1. Time course of the diffusion current. A: the diffusion currents computed with the monodoamin model at each node of the mesh. B: comparison between the mean value of the diffusion currents computed with the monodomain model and the fitted function $I_{\text {stim. }}$.

\section{Numerical results}

We compare the simulated numerical results of spiral waves obtained using the monodoamin model and the EK-CV model with the parametrization of the diffusion current (EK-CV-DIFF).We consider two different geometries: a $(5 \times 5 \times 0.1 \mathrm{~cm}) 3 \mathrm{D}$ tissue slab and an anatomically detailed bi-atrial domain. On all the test cases, we adopt the Mitchell-Schaeffer ionic model parameters shown in Table 1. The simulations of propagation on the bi-atrial geometry during $400 \mathrm{~ms}$ with the EK-CV-DIFF model required 21.89 seconds while the monodomain model required 1048.64 seconds on the same machine. We induce spiral waves in the simulations by applying S1-S2 protocol.

Table 1. Parameters of the MS ionic model.

\begin{tabular}{lcccc}
\hline$\tau_{\text {in }}$ & $\tau_{\text {out }}$ & $\tau_{\text {open }}$ & $\tau_{\text {close }}$ & $v_{\text {gate }}$ \\
& & & & \\
\hline $0.3 \mathrm{~ms}$ & $6 \mathrm{~ms}$ & $120 \mathrm{~ms}$ & $80 \mathrm{~ms}$ & 0.13 \\
\hline
\end{tabular}

\subsection{Tissue slab}

In this section, we show the simulation results obtained using the tissue slab geometry (dimensions $5 \times 5 \times 0.1 \mathrm{~cm}$ ). This was used to easily investigate the initiation of reentries and qualitatively compare the new approach to the actual 
state of the art. The number of vertices in the mesh is 20,490, the number of triangles is 15,256 and the number of tetrahedra is 99,420 with a characteristic mesh size $h=1.4 \mathrm{~mm}$. The conductivity of the tissue is equal to $0.001 \mathrm{~S} / \mathrm{cm}$. The tissue is initially at rest, we apply a first stimulus on the left edge with a duration of $t_{\text {stim }}$. The wave front then propagates from left to right of the tissue slab and we apply a second stimulus in a rectangular region $(x<3$ and $y<2.5)$ at time $S 2=300 \mathrm{~ms}$. Figure 2 shows the simulated action potentials at time $t=600 \mathrm{~ms}$, wtih the parametrised diffusive current (right) and with the constant expression adopted in [2](left). Both models generated a spiral wave with the same conduc-
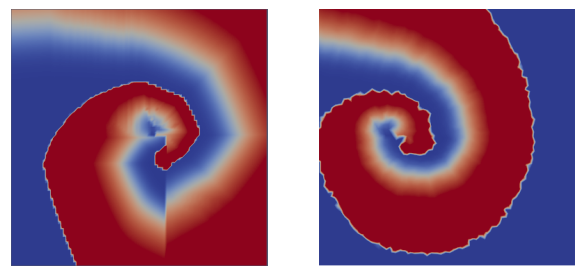

Fig. 2. Two snapshots of the transmembrane voltage $V_{m}$ at $t=600 \mathrm{~ms}$. On the left a constant stimulation current was applied and on the right we stimulate with the parameterised function of stimulus.

tion velocity. The EK-CV model, however, produced a strong discontinuity on the action potential in the depolarized region (Figure 2, left), not shown in the EK-CV-DIFF model (Figure 2, right). In that follows, we adopt the EK-CVDIFF model. Figure 3 shows the action potential at $t=(100,400,600,800 \mathrm{~ms})$, simulated with the EK-CV-DIFF model (top row) and with the monodomain MS model (bottom row). Both models initiate a stable spiral wave; the monodomain model, however, presents an activation front that propagates slower, when compared with the EK-CV-DIFF model. The slow propagation is caused by the coarse resolution $(h=1.4 \mathrm{~mm})$ here adopted, [8].

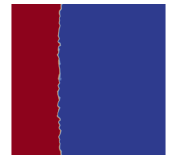

$\mathrm{T}=100 \mathrm{~ms}$

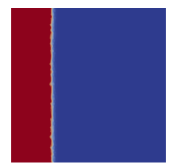

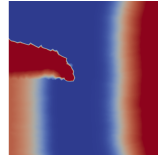

$\mathrm{T}=400 \mathrm{~ms}$

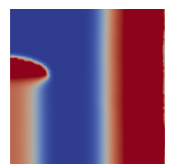

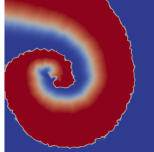

$\mathrm{T}=600 \mathrm{~ms}$

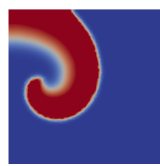

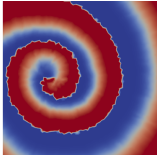

$\mathrm{T}=800 \mathrm{~ms}$

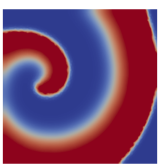

Fig. 3. Snapshots of the transmembrane voltage $V_{m}$ for the EK-CV-DIFF model (top row) and the monodomain model (bottom row) at different time steps. 


\subsection{Atrial geometry}

In this section, we present the simulation results obtained using the anatomical atrial geometry discretised here with a triangular surface mesh with 141,314 nodes and 275,515 elements. The conductivity of the tissue is equal to 0.09 $\mathrm{S} / \mathrm{cm}$. The tissue is initially at rest, we stimulate in the wall of the right atrium with an intensity $I_{\text {stim. }}$. A second stimulus is then applied at the bottom of the atrium $(y>21.1$ and $z<17.6)$ at time $S 2=240 \mathrm{~ms}$. In Fig.4, we show snapshots of the action potential at different times ( $t=10,250,390,430 \mathrm{~ms})$. In the top row of the figure, we show the obtained simulations with the EK-CVDIFF model. In the bottom row we present the simulation results obtained with the monodomain model. When comparing both simulations, we remark that two

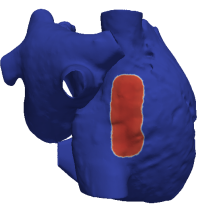

$\mathrm{T}=10 \mathrm{~ms}$

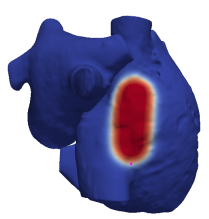

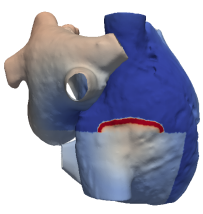

$\mathrm{T}=250 \mathrm{~ms}$

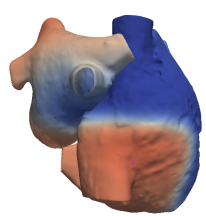

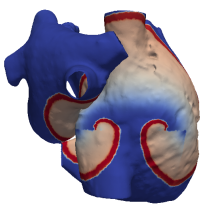

$\mathrm{T}=390 \mathrm{~ms}$

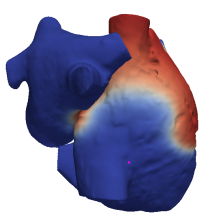

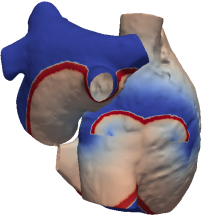

$\mathrm{T}=430 \mathrm{~ms}$

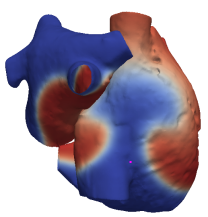

Fig. 4. Snapshots of the action potential $V_{m}$ of the EK-CV-DIFF model (top row) and the monodomain model (bottom row) at different time steps.

spiral waves occur in the same region for both models. We can also say that we have qualitatively the same pattern of reentry. On the other hand, we notice that the wave generated with the monodomain model is delayed with respect to the eikonal model: the pattern of the EK-CV-DIFF solution at time $390 \mathrm{~ms}$ corresponds to that for the monodomain solution at time $430 \mathrm{~ms}$.

\section{Discussion and conclusion}

In this work, we used the conduction velocity adapted eikonal model [2] in order to simulate reentry spiral waves. The new model that we present here combines the $\mathrm{CV}$-adapted eikonal model with a specific parametrized stimulation current obtained from the diffusion term of the monodomain model. The monodomain model is computed once off-line and the generated diffusion current has been approximated, parametrized and used to stimulate the MS ode system. The preliminary results showed that with this model we have been able to simulate 
spiral waves with the same pattern as the monodomain model. To the best of our knowledge, this is the first work able to generate spiral waves using the eikonal model. Differently from the monodomain model, [8], the EK-CV-DIFF formulation captures the correct value of the conduction velocity even using a coarse resolution. This eikonal model is easy to parametrize and thus could be a good candidate to conduct personalized simulations in order to simulate the electrical wave of a given patient. Our future work will be to parametrize this model in order to provide a new electrocardiography imaging tool especially in atrial fibrillation conditions.

\section{Acknowledgment}

This Project has received funding from the European Unions Horizon research and innovation programme under the Marie Skodowska-Curie grant agreement No. 860974 and by the French National Research Agency, grant references ANR10-IAHU04- LIRYC and ANR-11-EQPX-0030.

\section{References}

1. Clements, J.C., Nenonen, J., Li, P., Horáček, B.M.: Activation dynamics in anisotropic cardiac tissue via decoupling. Annals of biomedical engineering 32(7), 984-990 (2004)

2. Corrado, C., Zemzemi, N.: A conduction velocity adapted eikonal model for electrophysiology problems with re-excitability evaluation. Medical image analysis $\mathbf{4 3}$, 186-197 (2018)

3. Duchateau, J., Sacher, F., Pambrun, T., Derval, N., Chamorro-Servent, J., Denis, A., Ploux, S., Hocini, M., Jaïs, P., Bernus, O., et al.: Performance and limitations of noninvasive cardiac activation mapping. Heart Rhythm 16(3), 435-442 (2019)

4. Franzone, P.C., Guerri, L., Rovida, S.: Wavefront propagation in an activation model of the anisotropic cardiac tissue: asymptotic analysis and numerical simulations. Journal of mathematical biology 28(2), 121-176 (1990)

5. Mitchell, C.C., Schaeffer, D.G.: A two-current model for the dynamics of cardiac membrane. Bulletin of mathematical biology 65(5), 767-793 (2003)

6. Neic, A., Campos, F.O., Prassl, A.J., erer, S.A., Bishop, M.J., Vigmond, E.J., Plank, G.: Efficient computation of electrograms and ecgs in human whole heart simulations using a reaction-eikonal model. Journal of computational physics 346, 191-211 (2017)

7. Newville, M., Stensitzki, T., Allen, D.B., Rawlik, M., Ingargiola, A., Nelson, A.: Lmfit: Non-linear least-square minimization and curve-fitting for python. Astrophysics Source Code Library pp. ascl-1606 (2016)

8. Niederer, S.A., Kerfoot, E., Benson, A.P., Bernabeu, M.O.et.al.: Verification of cardiac tissue electrophysiology simulators using an n-version benchmark. Philosophical Transactions of the Royal Society A: Mathematical, Physical and Engineering Sciences 369(1954), 4331-4351 (2011)

9. Sethian, J.A.: A fast marching level set method for monotonically advancing fronts. Proceedings of the National Academy of Sciences 93(4), 1591-1595 (1996)

10. Wang, Y., Cuculich, P.S., Zhang, J., Desouza, K.A., Vijayakumar, R.et al..: Noninvasive electroanatomic mapping of human ventricular arrhythmias with electrocardiographic imaging. Science translational medicine 3(98), 98ra84-98ra84 (2011) 